\title{
SÍNDROMES DE DISPERSÃO DE DIÁSPOROS DE UM FRAGMENTO DE FLORESTA OMBRÓFILA MISTA EM TIJUCAS DO SUL, PR ${ }^{1}$
}

\author{
Diasporo Dispersion Syndromes in a Mixed Ombrophilous \\ Forest Fragment in Tijucas do Sul, PR
}

\author{
Dieter Liebsch \\ Biólogo, Pontifícia Universidade Católica do Paraná, Curitiba - PR. e-mail: dliebsch@bol.com.br \\ Luiz Antonio Acra \\ Professor, Pontifícia Universidade Católica do Paraná, Curitiba - PR. e-mail: laacra@rla01.pucpr.br
}

\begin{abstract}
Resumo
Este estudo foi realizado em um fragmento com 6 ha sob predomínio de Floresta Ombrófila Mista no Município de Tijucas do Sul - PR. Teve como objetivo registrar as síndromes de dispersão das espécies formadoras do sub-bosque desse fragmento. Para tanto, análises sobre as síndromes de dispersão foram efetuadas com observações no campo e em coletas mensais de outubro de 2001 a setembro de 2002. Foram identificadas 89 espécies e 54 gêneros, distribuídos em 32 famílias botânicas. A principal síndrome de dispersão foi a zoocoria com $65,1 \%$ das espécies presentes, $23,6 \%$ apresentaram a anemocoria como síndrome, seguida por $2,2 \%$ de autocoria e $9,0 \%$ não foram determinadas. O predomínio de espécies zoocóricas é importante na preservação e sucessão dos ambientes e, portanto, a eliminação desses dispersores pode comprometer a dinâmica desse fragmento florestal.
\end{abstract}

Palavras-chave: Floresta Ombrófila Mista; Síndromes de dispersão; Zoocoria; Anemocoria; Autocoria.

\begin{abstract}
This study was developed in a fragment with 6 ha of Mixed Ombrophilous Forest in Tijucas do Sul, Southern Municipal district of the Parana state. It had as aim to register the dispersion syndrome of the species in the sub-woodland of this fragment. The data and material was collected in field observations and monthly expeditions from October 2001 until September 2002. It was identified 89 species, 54 genera distributed among 32 botanical families. The main dispersion syndrome was the zoochoric, with $65.1 \%$ species; $23.6 \%$ of the species had the anemochoric syndrome, followed by $2.2 \%$ of autochoric and $9.0 \%$ were not determinate. The prevalence of the zoochoric species is important for the forest fragments preservation and succession; the elimination of this dispersers can compromise its dynamic.
\end{abstract}

Keywords: Mixed Ombrophilous Forest; Syndrome of dispersal; Zoochor; Anemochory; Autochory.

Financiado pelo CNPq/PELD.

Rev. Acad., Curitiba, v. 5, n. 2, p. 167-175, abr./jun. 2007 


\section{INTRODUÇÃO}

A Floresta Ombrófila Mista, segundo Gerhardt et al (2001) mais conhecida como Floresta de Araucária ou Pinheiral por apresentar uma presença massiva do pinheiro-do-paraná, pinheiro-brasileiro, Pinheiro ou simplesmente araucária (Araucaria angustifolia (Bert.) O. Kuntze), já revestiu grande parte do território paranaense, representando uma das mais ricas formações florestais do Estado, bem como do planalto sul-brasileiro (KOEHLER; PÉLLICO NETTO; SANQUETA, 1998; KLEIN, 1960, 1984; REITZ; KLEIN, 1966). Segundo Lindmann (1974), a araucária é a maior de todas as árvores florestais do sul do Brasil e devido à sua grandiosidade e expressiva dominância, tornou-se símbolo do emblema do Estado do Paraná (MAACK, 1968).

Atualmente, segundo Britez et al. (2000), resta menos de 1\% de Floresta com Araucária em boas condições de conservação no Estado do Paraná, sendo assim, a situação atual deste importante bioma é altamente crítica.

A dispersão é o deslocamento dos propágulos vegetais (frutos ou sementes) a partir da planta-mãe (HOWE; SMALWOOD, 1983). Esse é um dos processos mais importantes da regeneração natural de florestas tropicais, assim como da colonização de habitas (VAN DER PIJL, 1982). Essa colonização desempenha um papel fundamental no estabelecimento, desenvolvimento e evolução das espécies florestais, permitindo, assim, o intercâmbio de material genético dentro e fora das populações florestais (RONDON-NETO et al., 2001). A dispersão de sementes também é importante para a recuperação de áreas degradadas por atividades antrópicas, pois, para que se obtenham paisagens harmoniosas e auto-sustentáveis, é necessário que se considere a sucessão vegetal na recuperação dessas áreas (GRIFFITH et al., 1996).

A dispersão das sementes pode ser considerada como o procedimento que primariamente antecede à colonização de plantas, podendo assumir muita importância no entendimento da regeneração natural e das fases da sucessão secundária nas florestas (RONDON-NETO et al., 2001; MELO, 1997). Jansen (1970) propôs que a dispersão de sementes segue padrão inverso ao de recrutamento das plântulas. As chances de recrutamento próximo à planta-mãe podem ser muito baixas devido à competição e predação de sementes. À medida que as sementes se afastam da planta mãe, maior é a probabilidade de sobrevivência das plântulas (JANSEN, 1970; CIARK; CLARK, 1984). O manejo e a recuperação das florestas alteradas dependem da eficiência dos processos de dispersão dos propágulos e o estabelecimento das espécies de diferentes estágios sucessionais, sendo importante na manutenção da regeneração natural durante a dinâmica de sucessão da floresta.

O processo de dispersão, independente da forma de ocorrência, é muito complexo e envolve relações muito específicas entre plantas e agentes dispersores (FIGLIOLIA, 1993). Van der Pijl (1972) define essas síndromes como um conjunto de características, às vezes generalistas, às vezes restritas e precisas, que os propágulos apresentam, e que indicam o modo de dispersão de plantas.

Assim, como a dispersão é um meio para controlar e manter a diversidade biológica e, como tal, um pré-requisito para a continuidade dos ecossistemas tropicais, o estudo das relações planta/animal provou ser uma ferramenta útil para a análise da estrutura e o seu funcionamento e merece ser mais e melhor aproveitada.(MIKICH, 2001; MIKICH; SILVA, 2001).

Esse trabalho teve como objetivo investigar as síndromes de dispersão dos diásporos das espécies formadoras do sub-bosque de um fragmento de Floresta Ombrófila Mista Montana em Tijucas do Sul, colaborando para o entendimento da dinâmica desse fragmento, além de dar subsídios para o seu manejo e conservação.

\section{MATERIAIS E MÉTODOS}

\section{Área de estudo}

O estudo foi desenvolvido no Sistema Ecológico VIVAT FLORESTA, uma área com 5.084 ha, $\left(25^{\circ} 45 \mathrm{e} 26^{\circ} 00^{\prime} \mathrm{S} / 49^{\circ} 20 \mathrm{e} 49^{\circ} 05^{\prime} \mathrm{W}\right.$; alt. $\left.850-1350 \mathrm{~m}\right)$, situada no primeiro planalto paranaense, a cerca 
de 40-60 km ao sul da cidade de Curitiba, abrangendo parte dos municípios de São José dos Pinhais, Tijucas do Sul e Agudos do Sul (PARANÁ, 1987) (FIGURA 1).

O clima é do tipo subtropical úmido mesotérmico (Cfb) com ocorrência de geadas severas e verões frescos (IAPAR, 1978). A média das temperaturas dos meses mais quentes é inferior a $22^{\circ} \mathrm{C}$ e a dos meses mais frios é inferior a $18^{\circ} \mathrm{C}$. Precipitações médias anuais são de $1400 \mathrm{~mm}$ (FERREIRA, 1999).

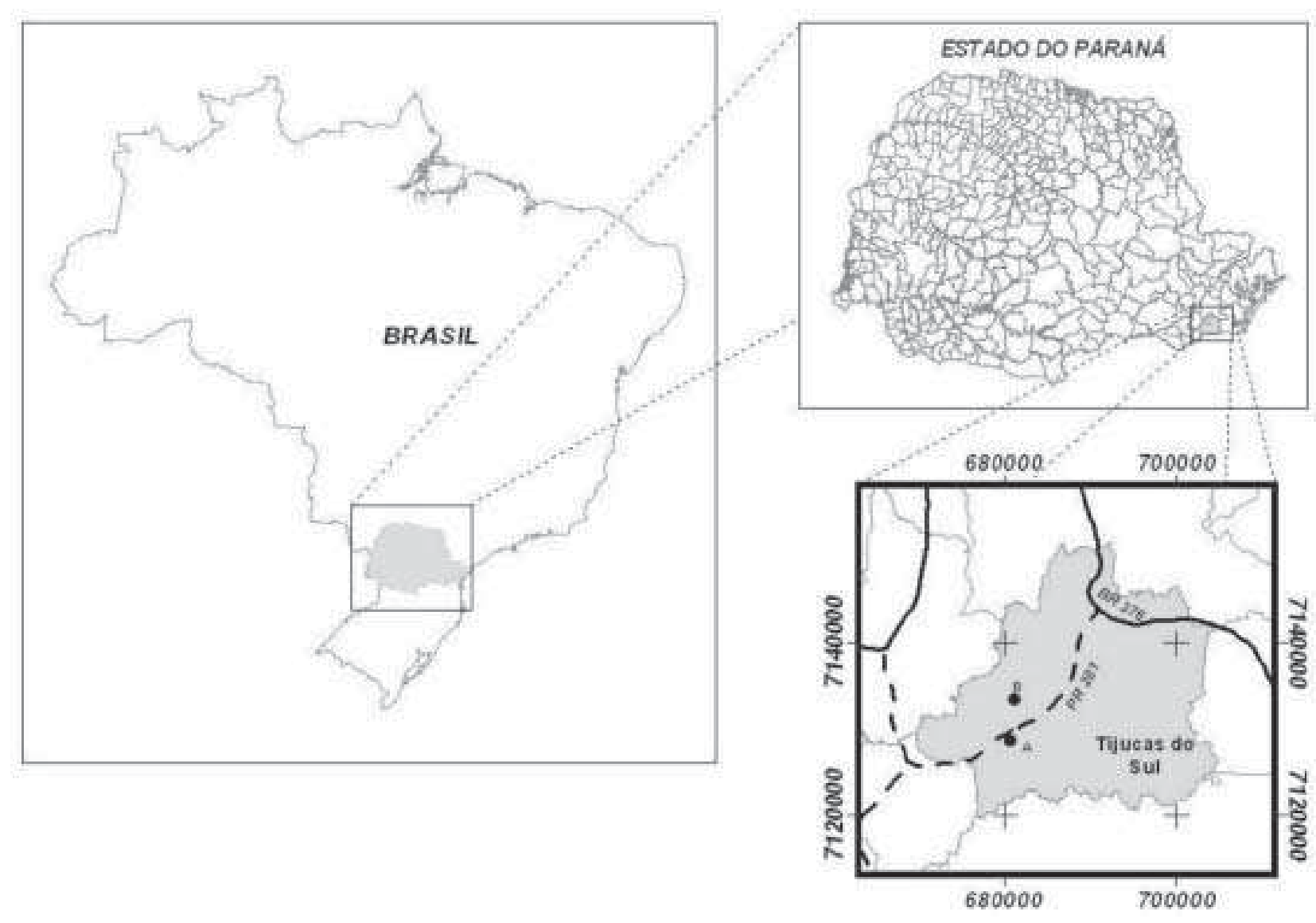

FIGURA 1 - Localização do município de Tijucas do Sul

Figure 1 - Location of the municipality of Tijucas do Sul

\section{Estabelecimento das unidades amostrais}

Para estudo da vegetação e seu gradiente de conservação, foram alocadas duas parcelas de 100 X $100 \mathrm{~m}$ (1 ha). A primeira parcela foi convencionada como área A. Nessa parcela, a vegetação primária foi muito alterada e a mata era utilizada como pastagem para o gado, que utilizava as ervas do sub-bosque como alimento complementar à pastagem do campo limpo (LIEBSCH; ACRA, 2002). Porém, há 7 anos não apresenta mais interferência, restando apenas algumas árvores da mata original e um denso subbosque, dando aspecto de capoeirinha e capoeira, segundo classificação do IBGE (1992). A segunda parcela foi chamada de área $\mathrm{B}$, localizada no interior da floresta, onde a intervenção ficou restrita à retirada seletiva de madeira e a algumas trilhas de acesso à floresta, e apresenta a estratificação evidente, mantendo características fisionômicas de Floresta Ombrófila Mista (LIEBSCH, 2002). 


\section{Análise das síndromes}

As espécies encontradas foram agrupadas quanto às síndromes de dispersão, com base na classificação de Van der Pijl (1972), em três categorias: 1- anemocóricas: quando os diásporos apresentam-se alados, plumosos ou em forma de balão ou poeira; 2- zoocóricas: quando apresentam atrativos e/ou fontes alimentares em seus diásporos, e também aquelas com estruturas adesivas como ganchos, cerdas, espinhos, etc.; 3- autocóricas: quando não se encaixaram nas duas categorias anteriores, ficando nesta categoria as espécies barocóricas e aquelas com dispersão explosiva. Em alguns casos, não foi possível determinar a síndrome do diásporo por deficiência nas coletas de dados, uma vez que as coletas eram realizadas mensalmente.

\section{RESULTADOS}

Considerando-se ambas as áreas, foram encontradas 65,1\% de espécies que apresentam diásporos zoocóricos, 23,6\% anemocóricos, 2,2\% autocóricos e 9,0\% de plantas com síndrome não determinada (FIGURA 2). Quando essa observação é feita para cada uma das áreas estudadas, foi encontrada na área A 63,1\% dos frutos/sementes, sendo zoocóricos, que estão representados principalmente pelas famílias Solanaceae, Melastomataceae e Myrtaceae. A anemocoria foi observada com distribuição exclusiva na capoeira e é representada por $25 \%$ das espécies. A principal representante desta síndrome foi a família Asteraceae, a qual apresenta 100\% dos frutos anemocóricos na área estudada (TABELA 1). A autocoria foi observada em apenas 2,3\% dos indivíduos e também exclusivamente na capoeira (TABELA 1).

Para a área B, a zoocoria está presente em 100\% dos frutos observados, principalmente com a família Myrtaceae (TABELA 1).

Em muitos casos, não foi possível determinar que síndrome o fruto apresentou, sendo nesse caso denominado "não determinado". Isso ocorreu com $9 \%$ das espécies para ambas as áreas.

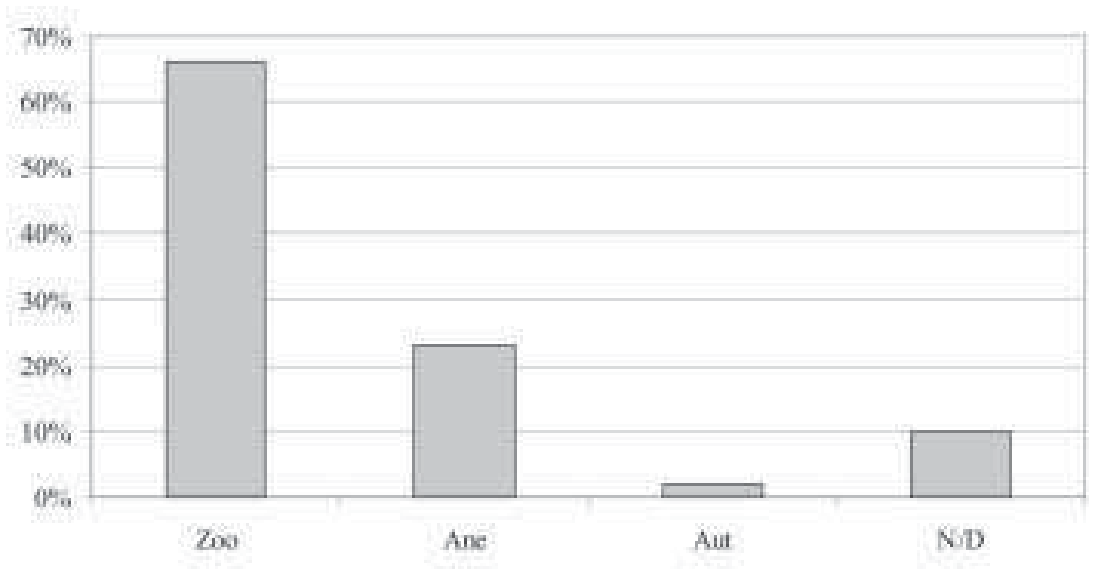

FIGURA 2 - Distribuição das síndromes de dispersão dos diásporos em dois fragmentos de Floresta Ombrófila Mista em Tijucas do Sul, Paraná

Figura 2 - Diasporo Dispersion Syndromes Distribution in two Mixed Ombrophilous Forest fragments in Tijucas do Sul, Parana 
Síndromes de dispersão de diásporos de um fragmento de Floresta Ombrófila Mista em Tijucas do Sul, PR 171

TABELA 1 - Distribuição das espécies coletadas em Tijucas do Sul (área A capoeira, área B Floresta) Table 1 -Distribution of the collected species in the municipality of Tijucas do Sul (area A scrub, area B forest)

\begin{tabular}{|c|c|c|c|c|}
\hline Família & Espécie & Área A & Área B & Sindrome \\
\hline Asclepiadaceae & Asclepias curassavica $\mathrm{L}$. & $\mathrm{X}$ & & Anemocoria \\
\hline \multirow[t]{14}{*}{ Asteraceae } & Austroetupatorim pictuatum (Malme) R. M. King \& H. Robinson & $\mathrm{X}$ & & Anemocoria \\
\hline & $\begin{array}{l}\text { Bacharis brachylaenoides DC. Var. polycephala (Sch. Bip) G. M. } \\
\text { Barroso }\end{array}$ & $\mathrm{X}$ & & Anemocoria \\
\hline & Baccharis trimera (Less) DC. & $\mathrm{X}$ & & Anemocoria \\
\hline & Baccharidastrum triplinerve (Less) Cabrera & $\mathrm{x}$ & & Anemocoria \\
\hline & Chaptalia nutans (L.) Polak. & $\mathrm{X}$ & & Anemocoria \\
\hline & Eupatorium tweedianum Hook. Et Arn. & $\mathrm{x}$ & & Anemocoria \\
\hline & Eupatorium sp & $\mathrm{x}$ & & Anemocoria \\
\hline & Jungia selowï Less & $\mathrm{X}$ & & Anemocoria \\
\hline & Mikania orleansensis Hieron. & $\mathrm{x}$ & & Anemocoria \\
\hline & Podocoma notobellidiastrum (Griseb) Nesom & $\mathrm{X}$ & & Anemocoria \\
\hline & Senecio icoglossus DC: & $\mathrm{x}$ & & Anemocoria \\
\hline & Vernonia florida gardner & $\mathrm{x}$ & & Anemocoria \\
\hline & Vernonia polianthes Less & $\mathrm{x}$ & & Anemocoria \\
\hline & Vernonia westiniana Less & $\mathrm{X}$ & & Anemocoria \\
\hline Anacardiaceae & Schinus terebinthifolius Raddi & $\mathrm{X}$ & & Zoocoria \\
\hline Balsaminaceae & Impatiens walleriana Hook & $\mathrm{X}$ & & Autocoria \\
\hline Berbiridaceae & Berberis laurina Billb. & $\mathrm{x}$ & & Zoocoria \\
\hline Bignoniaceae & Jacaranda puberula Cham. & $\mathrm{x}$ & & Anemocoria \\
\hline Budlejaceac & Budlejam brasiliensis Jacq. & $\mathrm{x}$ & & Anemocoria \\
\hline Caesalpinaceae & Senna $s p$ & $\mathrm{x}$ & & Autocoria \\
\hline \multirow[t]{2}{*}{ Commelinaceae } & Commelina erecta $\mathrm{L}$. & $\mathrm{x}$ & & ND \\
\hline & Tradechantia sp & $\mathrm{X}$ & & ND \\
\hline Cyperaceae & Fimbristylis autunalis (L.) Roem \& Schult. & $\mathrm{x}$ & & Anemocoria \\
\hline \multirow[t]{2}{*}{ Euphorbiaceae } & Croton ceanothipholius Bail. & $\mathrm{X}$ & & ND \\
\hline & Croton celtidifolitus Bail. & & $\mathrm{x}$ & Zoocoria \\
\hline Flacourtiaceae & Casearia sylvestris Sw. & $\mathrm{x}$ & $\mathrm{X}$ & Zoocoria \\
\hline \multirow[t]{3}{*}{ Lamiaceae } & Hyptis lappulaceae Mart ex Benth & $\mathrm{X}$ & & ND \\
\hline & Salvia meliceflora Benth. & $\mathrm{x}$ & & ND \\
\hline & Ocimum selloi Benth. & $\mathrm{X}$ & & ND \\
\hline Lythraceae & Heimia myrtifolia C. DC. & $\mathrm{X}$ & & ND \\
\hline \multirow[t]{2}{*}{ Malvaceae } & Pavonia sepium A. St. Hil. & $\mathrm{x}$ & & Zoocoria \\
\hline & Sida potentilloides St. Hil. & $\mathrm{x}$ & & Zoocoria \\
\hline Moraceae & Ficus luschnathiana (Miq.) Miq. & $\mathrm{X}$ & $\mathrm{X}$ & Zoocoria \\
\hline \multirow[t]{13}{*}{ Melastomataceae } & Leandra australis (Cham.) Cogn. & $\mathrm{x}$ & $\mathrm{x}$ & Zoocoria \\
\hline & Leandra refracta Cogn. & $\mathrm{X}$ & $\mathrm{x}$ & Zoocoria \\
\hline & Leandra regnellii (Triana) Cogn. & $\mathrm{x}$ & $\mathrm{x}$ & Zoocoria \\
\hline & Leandra sy/vestris DC. & $\mathrm{x}$ & $\mathrm{x}$ & Zoocoria \\
\hline & Leandra xanthocoma (Naud.) Cogn. & $\mathrm{X}$ & & Zoocoria \\
\hline & Leandra sp I & $\mathrm{x}$ & & Zoocoria \\
\hline & Leandra sp 2 & $\mathrm{X}$ & & Zoocoria \\
\hline & Leandra sp 3 & $\mathrm{x}$ & & Zoocoria \\
\hline & Leandra sp 4 & $\mathrm{x}$ & & Zoocoria \\
\hline & Miconia yemalis Naud. & $\mathrm{X}$ & & Zoocoria \\
\hline & Miconia petropolitana $\operatorname{Cog} n$ & $\mathrm{x}$ & $\mathrm{x}$ & Zoocoria \\
\hline & Miconia theaezans (Bompl.) Cogn. & $\mathrm{x}$ & $\mathrm{x}$ & Zoocoria \\
\hline & Tibouchina cerastifolia (Naud.) Cogn. & $\mathrm{x}$ & & Anemocoria \\
\hline Meliaceac & Cabralea canjerana (Vell.) Mart. & & $\mathrm{x}$ & Zoocoria \\
\hline
\end{tabular}

Rev. Acad., Curitiba, v. 5, n. 2, p. 167-175, abr./jun. 2007 
TABELA 1 - Distribuição das espécies coletadas em Tijucas do Sul (área A capoeira, área B Floresta) Table 1 - Distribution of the collected species in the municipality of Tijucas do Sul (area A scrub, area B forest)

(Continuação)

(Continuation)

\begin{tabular}{|c|c|c|c|c|}
\hline Myrtaceae & Eugenea neovernicosa Sobral & & $\mathrm{X}$ & Zoocoria \\
\hline & Gomidesia selowiana $\mathrm{Berg}$. & $\mathrm{X}$ & & Zoocoria \\
\hline & Myrceugenia cucullata Legrand. & $\mathrm{X}$ & $\mathrm{X}$ & Zoocoria \\
\hline & Myrceugenia euosma (Berg.) Legrand. & $\mathrm{X}$ & & Zoocoria \\
\hline & Myrceugenia sp 1 & $\mathrm{X}$ & $\mathrm{X}$ & Zoocoria \\
\hline & Myrceugenia sp 2 & $\mathrm{X}$ & $\mathrm{X}$ & Zoocoria \\
\hline & Mynceugenia sp 3 & $\mathrm{X}$ & & Zoocoria \\
\hline & Myrtaceae 1 & $\mathrm{X}$ & $\mathrm{X}$ & Zoocoria \\
\hline Orchidaceae & Orchidaceae 1 & $\mathrm{X}$ & & Anemocoria \\
\hline \multirow[t]{2}{*}{ Piperaceae } & Piper gaudichaudianum kunth. & $\mathrm{X}$ & & Zoocoria \\
\hline & Piper xilloteoides (Kunth.) Steud. & $\mathrm{X}$ & $\mathrm{X}$ & Zoocoria \\
\hline \multirow[t]{3}{*}{ Poaceae } & Ichnanthus sp & $\mathrm{X}$ & & Anemocoria \\
\hline & Panicum maximum Jacq. & $\mathrm{X}$ & $\mathrm{X}$ & Zoocoria \\
\hline & Setaria sp & $\mathrm{X}$ & & Zoocoria \\
\hline Polygalaceae & Polygala lancīfolia A. St. Hil. & $\mathrm{X}$ & & ND \\
\hline \multirow[t]{2}{*}{ Rosaceae } & Rubus brasiliensis Mart. & $\mathrm{X}$ & & Zoocoria \\
\hline & Rubus sellowii Koehne & $\mathrm{X}$ & & Zoocoria \\
\hline \multirow[t]{2}{*}{ Rubiaceae } & Psychotria stachyoides Benth. & $\mathrm{X}$ & & Zoocoria \\
\hline & Psychotria suterella Müll. Arg. & & $\mathrm{X}$ & Zoocoria \\
\hline Sapindaceae & Allophyllus edulis (A. St. - Hil.) Radlk. & $\mathrm{X}$ & & Zoocoria \\
\hline \multirow[t]{16}{*}{ Solanaceae } & Athenaea picta (Mart.) Sendtn. & $\mathrm{X}$ & & Zoocoria \\
\hline & Aureliana fasciculata (Vell.) Sendth. var & & & \\
\hline & Aureliana longifolia (Sendth) Hunz \& Barbosa & $\mathrm{X}$ & $\mathrm{X}$ & Zoocoria \\
\hline & Brunfelsia brasiliensis (Spreng) Smith \& Downs & $\mathrm{X}$ & $\mathrm{X}$ & Zoocoria \\
\hline & Cestrum amictum Schltdl. & $\mathrm{X}$ & & Zoocoria \\
\hline & Cyphomandra kleinit Smith \& Downs & $\mathrm{X}$ & & Zoocoria \\
\hline & Cyphomandra macrophylla Smith \& Downs & $\mathrm{X}$ & & Zoocoria \\
\hline & Solamum acenosum Sendth. & $\mathrm{X}$ & $\mathrm{X}$ & Zoocoria \\
\hline & Solanum concinum Schott Ex Sendth & $\mathrm{X}$ & & Zoocoria \\
\hline & Solanum diflorum Vell. & $\mathrm{X}$ & $\mathrm{X}$ & Zoocoria \\
\hline & Solanum johanae Bitter & $\mathrm{X}$ & $\mathrm{X}$ & Zoocoria \\
\hline & Solanum lacerdae Dusén & $\mathrm{X}$ & $\mathrm{X}$ & Zoocoria \\
\hline & Solamum ranulatum Sendth & $\mathrm{X}$ & & Zoocoria \\
\hline & Solanum reflexum Schrank & $\mathrm{X}$ & & Zoocoria \\
\hline & Solanum schwackeanum Smith \& Downs & $\mathrm{X}$ & & Zoocoria \\
\hline & Solanum Sp & $\mathrm{X}$ & & Zoocoria \\
\hline Tiliaceae & Triumpheta semitriloba Jacq. & & $\mathrm{X}$ & Zoocoria \\
\hline Thymelaeceae & Daphnopsis racemosa Griseb. & $\mathrm{X}$ & & Zoocoria \\
\hline \multirow[t]{2}{*}{ Verbenaceae } & Lantana brasiliensis Link & $\mathrm{X}$ & $\mathrm{X}$ & Zoocoria \\
\hline & Lantana fucata Lindl. & $\mathrm{X}$ & & Zoocoria \\
\hline Winteraceae & Drymis brasiliensis Miers. & $\mathrm{X}$ & $\mathrm{X}$ & Zoocoria \\
\hline Zingberaceae & Hedychium coronarium Koen & $\mathrm{X}$ & & Zoocoria \\
\hline
\end{tabular}




\section{DISCUSSÃO}

Os dados encontrados corroboram as informações de Rondon-Neto et al. (2001), também na Floresta Ombrófila Mista, mas esses autores utilizaram o estrato arbóreo, encontrando 75,7\% de espécies zoocóricas, 18,9\% anemocóricas e 5,4\% autocóricas. Carmo e Moreliato (2001) encontraram ao longo da Bacia do Rio Tibagi um total de 261 espécies, sendo 67,8\% zoocóricas, 15,7\% anemocóricas e 7,7\% das espécies apresentam outros modos de dispersão. Mikich e Silva (2001) encontraram 61\% das espécies zoocóricas em uma Floresta Estacional Semidecidual.

Estudos realizados em florestas tropicais úmidas mostraram que a proporção de espécies anemocóricas foi inferior a 20\% e a proporção de zoocóricas superior a 80\% (MORELIATO; LEITÃOFILHO, 1992; TALORA; MOREILATO, 2000). Apesar de o presente estudo estar restrito ao estrato inferior da floresta, os valores aqui observados foram semelhantes aos anteriormente citados.

Batalha e Mantovani (2000) realizaram um estudo sobre os padrões fenológicos do cerrado na região sudeste do Brasil e constataram que no estrato herbáceo 38,07\% das espécies são anemocóricas, $36,98 \%$ autocóricas e 25,95\% são zoocóricas. No estrato arbóreo, 25,92\% são espécies anemocóricas, $12,04 \%$ autocóricas e, a maioria, 62,04\%, são zoocóricas. Esses valores estão de acordo com Gentry (1982), que observou que, nas florestas neotropicais, a proporção de espécies zoocóricas diminui das áreas úmidas em direção às áreas secas. Esse padrão foi observado no presente estudo, áreas abertas à proporção de espécies anemocóricas é maior comparada com áreas florestais. Motta-Junior e Lombardi (2002) realizaram um levantamento bibliográfico sobre a ocorrência de zoocoria nas florestas de galeria do Complexo do Cerrado e constataram que, em todos os estudos (14 no total), a zoocoria foi o principal modo de dispersão, variando de $62,6 \%$ a $89,4 \%$ das espécies.

A família Asteraceae e as outras espécies que apresentam como síndrome de dispersão a anemocoria foram encontradas exclusivamente na capoeira, ou seja, formações abertas, corroborando a afirmação de Howe e Smallwood, (1983) de que a falta de um dossel contínuo favorece as espécies com dispersão pelo vento.

Segundo Fenner (1985), Mikich (2001) e Mikich e Silva (2001), nas formações florestas em estádios sucessionais mais avançados, a complexidade da comunidade vegetal aumenta, atraindo aves e mamíferos. Esse processo pode ser observado no presente estudo, pois os indivíduos das famílias Myrtaceae, Melastomataceae (com exceção de Tibouchina) e Solanaceae são zoocóricas. Juntas, essas famílias contribuem com 35 espécies do total 89 espécies. Tal fato pode ter um papel importante no incremento em riqueza específica e complexidade estrutural destas áreas, uma vez que a presença de espécies zoocóricas pode atrair potenciais dispersores de espécies características destes ambientes, ou até mesmo de etapas subseqüentes da sucessão, e que eventualmente não ocorram nestes locais (MIKICH, 2001; MIKICH; SILVA, 2001).

\section{CONCLUSÕES}

No presente fragmento, o predomínio de espécies zoocóricas tem grande importância, pois a entrada de propágulos zoocóricos está ligada diretamente à fauna. A manutenção da fauna e a exclusão dos distúrbios antrópicos são de extrema importância para a dinâmica nesse fragmento florestal. A anemocoria está relacionada à área de capoeira, onde predominam espécies pioneiras, pois áreas abertas favorecem diásporos que utilizam o vento como dispersor. 


\section{AGRADECIMENTOS}

Os autores agradecem ao Professor Dr. Sylvio Péllico Netto, pela viabilidade do auxílio financeiro. A Arthur Ângelo Bispo de Oliveira, Rodrigo Nápoli, pela correção do original. A Carlyle Santim Sguassabia, Daniel Carvalho Carneiro e Daniel Isolani, pelo auxílio nas coletas. Ao Engenheiro Florestal M. Sc. Alexandre Bernardi Koheler è à Bióloga M. Sc. Marília Borgo. Ao Dr. Gert G. Hatschbach, ao Botânico Osmar dos Santos Ribas e ao Prof. Dr. Renato Goldenberg, pela identificação de alguns exemplares botânicos.

\section{REFERÊNCIAS}

BATALHA, M. A.; MANTOVANI, W. Reprodutive phenological patterns of cerrado plant espécies at the Pé-de-Gigante Reserve (Santa Rita do Passa Quatro, SP, Brasil): a comparison between the herbaceous and woody floras. Rev. Brasil Biol. São Paulo, v. 60, n. 1, p. 129-145, 2000.

BRITEZ, R. M. et al. Estratégias de conservação da Floresta de Araucária para o Estado do Paraná Diagnóstico da vegetação. In: CONGRESSO BRASILEIRO DE UNIDADES DE CONSERVAÇÃO, 11, 2000, Campo Grande. Anais... Campo Grande, 2000. p. 731-737.

CARMO, M. R. B.; MORELLATO, L. P. C. Fenologia de árvores e arbustos das matas ciliares da Bacia do Rio Tibagi, estado do Paraná, Brasil. In: RODRIGUES, R. R.; LEITAO FILHO, H. F. (Org.). Matas ciliares: conservação e recuperação. Campinas: UNICAMP/FAPESP, 2001. p. 125-141.

CLARK, D. A.; CLARK, D. B. Spacing dynamics of a tropical tree: evaluation of the Janzen-Connell model. American Naturalist. Chicago, v. 12, p. 769-788, 1984.

FENNER, M. Seed ecology. London: Chapman and Hall, 1985.

FERREIRA, J. C. V. O Paraná e seus municípios. 2. ed. Cuiabá: Memória do Brasil, 1999.

FIGLiOOLIA, M. B. Maturação de sementes de Inga uruguensis Hook et Arn. Associada à fenologia reprodutiva e a dispersão de sementes em floresta ripária do rio Mogi Guaçu - município de Mogi Guaçu. 1993. 150 f. Dissertação (Mestrado em Engenharia Florestal) - Escola Superior de Agricultura Luiz de Queiroz, Universidade de São Paulo, 1993.

GENTRY, A. H. Patterns of neotropical plant species diversity. Evolution Biology, v. 15. p. 1-84, 1982.

GERHARDT, E. J. et al. Contribuição da análise multivariada, na classificação de sítios em povoamentos de Araucaria angustifólia (Bert.) O. Ktze., baseada nos fatores físicos e morfológicos do solo e no conteúdo de nutrientes da serrapilheira. Ciência Florestal, Santa Maria, v. 11, n. 2, p. 41-57, 2001.

GRIFFITH, J. J.; DIAS, L. E.; JUCKSCH, I. Recuperação de áreas degradadas usando vegetação nativa. Saneamento Ambiental, n. 37, p. 28-37, 1996.

HOWE, H. F.; SMALLWOOD, J. Ecology of seed dispersal. Annual Review of Ecology and Systematics, n. 13, p. 201-228, 1983.

IAPAR. Cartas Climáticas Básicas do Estado do Paraná. Londrina: Fundação Instituto Agronômico do Paraná, 1978, 38 p.

IBGE. Manual técnico da vegetação brasileira. Rio de Janeiro: IBGE, 1992. p. 92. (Série Manuais Técnicos em Biociências, v. 1).

JANZEN, D. H. Herbivores and the number of the tree species in tropical forest. Am. Nat, v. 104, p. 501-528, 1970.

KLEIN, R. M. O aspecto dinâmico do pinheiro brasileiro. Sellowia, Itajaí, v. 12, n. 12, p. 17-44. 1960. 
. Aspectos dinâmicos da vegetação do sul do Brasil. Sellowia, Itajaí, v. 36, n. 36, p. 5-54, 1984.

KOEHLER, A.; PÉLLICO NETTO, S.; SANQUETA, C. R. Análise da estrutura de uma Floresta Ombrófila Mista semidevastada, Fazenda Gralha Azul, Região Metropolitana de Curitiba, com implicações ao manejo. Revista Acadêmica, Curitiba, v. 9, n. 1, p. 37-60, 1998.

LIEBSCH, D. Levantamento da vegetação de sub-bosque de um remanescente de floresta Ombrófila mista em Tijucas do Sul, Paraná, Brasil. Curitiba. 2002. 40 f. Monografia (Curso de Biologia) - Pontifícia Universidade Católica do Paraná, Curitiba, 2002.

.; ACRA. L. A. 2002. Asteraceae Dumorter (=Compositae Giseke) de um Capão de Floresta Ombrófila Mista, da Area do PROAÇAO/PUCPR, Município de Tijucas do Sul, Paraná, Brasil. In: BIOCEC, III., 2002, Curitiba. Anais... Curitiba, 2002, p. 25.

LINDMANN, C. A. A vegetação no Rio Grande do Sul. Belo Horizonte: EDUSP, 1974.

MAACK. R. Geografia física do estado do Paraná. Curitiba: José Olympio, 1968.

MELO, V. A. Poleiros artificiais e dispersão de sementes por aves em uma área de reflorestamento, no estado de Minas Gerais. Viçosa. 1997. 39 f. Dissertação (Mestrado em Ciência Florestal) Universidade Federal de Viçosa, Minas Gerais, 1997.

MIKICH, S. B. Frugivoria e dispersão de sementes em uma pequena reserva isolada do Estado do Paraná, Brasil. Curitiba, 2001. 145 f. Tese (Doutorado em Zoologia) - Universidade Federal do Paraná, Curitiba, 2001.

.; SILVA, S. M. Composição florística e fenologia das espécies zoocóricas de remanescentes de Floresta Estacional Semidecidual no centro-oeste do Paraná, Brasil. Acta Bot. Bras., Rio de Janeiro, v. 15, n. 1, p. 89-113, 2001.

MORELLATO, L. P. C.; LEITÃO-FILHO, H. F. Padrões de frutificação e dispersão na serra do Japi. In: MORELLATO, L. P. C. (Org.). História natural da serra do Japi: ecologia e preservação de uma área florestal no sudeste do Brasil, Campinas: UNICAMP, p. 112-140, 1992.

MOTTA-JUNIOR, J. C.; LOMBARDI, J. A. Ocorrência de zoocoria em florestas-de- galeria no Complexo do Cerrado, Brasil. Biotemas, Florianópolis, v. 15, n. 1, p. 59-81, 2002.

PARANÁ. Atlas do estado do Paraná. Curitiba: Instituto de Terras, Cartografias e Florestas, 1987. p. 73.

REITZ, R.; KLEIN, R. M. Araucariáceas. Flora ilustrada Catarinense. Itajaí: Herbário Barbosa Rodrigues, 1966. 63 p.

RONDON-NETO, R. M.; WATZLAWICK, L. F.; CALDEIRA, M. V. W. Diversidade florística e síndromes de dispersão de diásporos das espécies arbóreas de um fragmento de floresta ombrófila mista. Revista Ciências Exatas e Naturais, Guarapuava, v. 3, n. 2, p. 209-216, 2001.

TÁLORA, D. C.; MORELLATO, L. P. C. Fenologia de espécies arbóreas em floresta de planície litorânea do sudeste do Brasil. Rev Bras Bot. São Paulo, v. 23, n. 1, p. 13-26, 2000.

VAN DER PIJL, L. Principles of dispersal in higher plants. 2. ed. Berlim: Springer-Verlag, 1972. Principles of dispersal in higher plants. 3. ed. New York: Springer-Verlag, 1982.

Recebido em: 25/06/2006

Received in: 06/25/2006

Aprovado em: 30/09/2006

Accepted in: 09/30/2006 